\title{
Diaschisis and Neurobehavior
}

\author{
Dang Khoa Nguyen and M.I. Botez
}

\begin{abstract}
We review forms of diaschisis and their relationship with neurobehavioral and neuropsychological findings. The following forms of diaschisis are discussed: (1) cortico-cerebellar diaschisis; (2) cerebello-cortical diaschisis; (3) transhemispheric diaschisis; (4) cortico-thalamic diaschisis; (5) thalamo-cortical diaschisis; and (6) basal ganglia-cortical diaschisis. For each form, the neurobehavioral and neuropsychological findings are discussed. Diaschisis can be classified from the behavioral point of view as follows: (1) forms in which the metabolic effect at distance is not followed by neurobehavioral impairment; (2) forms in which the remote metabolic impairment could induce neurobehavioral and neuropsychological disorders; and (3) forms in which the role of the lesion itself and its remote metabolic effects on the production of neurobehavioral and neuropsychological abnormalities cannot be disentangled.
\end{abstract}

RÉSUMÉ: Diaschisis et comportement: Faire une revue concernant les diverses formes de diaschisis et leurs relations avec les données comportementales et neuropsychologiques. Les auteurs présentent les différentes formes de diaschisis: (1) diaschisis cortico-cérébelleaux; (2) diaschisis cérébello-cortical; (3) diaschisis transhémisphérique; (4) diaschisis cortico-thalamique; (5) diaschisis thalamo-cortical; et (6) diaschisis des ganglions de la base - cortex cérébral. On discute de la présence ou de l'absence des troubles neuropsychologiques et du comportement moteur et non-moteur pour chaque forme de diaschisis. Du point de vue des troubles neuropsychologiques et des troubles du comportenent moteur et non-moteur, les diaschisis peuvent être classifiés comme suit: (1) une forme de diaschisis non accompagnée par des troubles de comportement ou des troubles neuropsychologiques; (2) une forme de diaschisis dans laquelle la souffrance métabolique à distance puet déterminer des troubles de comportement et des troubles neuropsychologiques; et enfin (3) une forme de diaschisis dans laquelle les effets de la lésion primaire et des effets métaboliques à distance ne peuvent pas être différenciés.

Can. J. Neurol. Sci. 1998; 25: 5-12

\author{
ABBREviations \\ ACA: anterior cerebral artery \\ $C B F$ : cerebral blood flow \\ CeBF: cerebellar blood flow \\ $C C D$ : crossed cerebellar diaschisis \\ $C M R_{g l c}:$ mean cerebral glucose utilization \\ CNS: central nervous system \\ $C T$ : computed tomography \\ CVA: cerebrovascular accident \\ ECT: emission computed tomography \\ EEG: electroencephalogram \\ ${ }^{18} \mathrm{FCH}_{3}$ : fluorine ${ }^{18}$ fluoromethane \\ ${ }^{18} \mathrm{FDG}:{ }^{18} \mathrm{~F}$-fluorodeoxyglucose \\ $99 m$ Tc-HMPAO: Technetium-99m hexamethyl- \\ propyleneamineoxime \\ 123/-IMP: lodine-123 iodoamphetamine \\ $L C M R_{\text {glc }}$ : local cerebral glucose utilization \\ MCA: middle cerebral artery \\ MRI: magnetic resonance imaging \\ ${ }^{13} \mathrm{NH}_{3}:{ }^{13} \mathrm{~N}$-ammonia \\ PET: positron emission tomography \\ SPECT: single photon emission computed tomography \\ $V L$ : nucleus ventralis \\ Vim: nucleus ventralis intermedius
}

Diaschisis (di-as'kî-sis), derived from the Greek "dia- + schizein" meaning a splitting, is classically defined as a sudden inhibition of function produced by an acute focal disturbance in an anatomically intact portion of the brain remote from the original seat of injury, but anatomically connected with it through fiber tracts.' It is well known now that these changes at a distance may also be the result of not only acute lesions but also chronic degenerative ones.

This phenomenon was first described in 1914 by the Russian neurologist Von Monakow ${ }^{2}$ who introduced the terms: "diaschisis cortico-spinalis", i.e., impaired function of spinal motor neurons due to cortical motor lesions; "diaschisis associativa", i.e., cortical dysfunction due to lesions of connected areas within one hemisphere; and "diaschisis commisuralis", i.e., cortical dysfunction due to lesions of the interconnected contralateral hemisphere. As a matter of fact, diaschisis was first described as an explanation for spinal shock after spinal cord transection. ${ }^{2}$

In day-to-day clinical neurology, one knows that symptoms of CNS lesions, especially tumors, may be due to the lesions

From the Behavioral Neurology. Neurobiology and Neuropsychology Research Unit and Neurology Service, Hotel-Dieu Hospital and University of Montreal. Montreal. RECEIVED APRIL 28, 1997. ACCEPTED IN FINAL FORM ALGUST 7, 1997.

Reprint requests to: M.I. Botez, Hôtel-Dieu Hospital, 3840 rue St-Urbain. Montreal. Québec, Canada H2W 1 T8 
themselves, to edema surrounding the lesions but also to distance effects. This phenomenon is also observed in acute spinal cord injuries where spinal shock, characterized by areflexia, loss of sensation and flaccid paralysis below the level of the lesion, a flaccid bladder and a lax anal sphincter, may occur. These patients may regain substantial or even normal neurological function, even though the initial neurological deficit may be severe. $^{3}$

The term "diaschisis" has been extended to include all effects at a distance from acute and chronic lesions of the CNS resulting in metabolism and perfusion alterations remote from the primary site of lesion and hence, depression of neuronal function.

The term diaschisis presupposes an anatomic lesion as shown by standard CT scans or MRI and metabolic and perfusion disorders on both the lesion site as well as in structures remote from the primary lesion.

Modern neuroimaging techniques (PET, SPECT) have allowed further understanding of the diaschisis phenomenon, establishing, for example, that the clinical neurological manifestations of an acute stroke all far exceed the size of the infarct, and that, furthermore, local cerebral metabolism and local CBF are measurably reduced in areas of the brain far localized from the infarct locus (areas which appear normal on standard cerebral CT or MRIs), confirming Von Monakow's theories.

At least 6 types of diaschisis have now been described and more will probably be found.

\section{FORMS OF DIASCHISIS}

\section{Cortico-cerebellar Diaschisis (or Crossed Cerebellar Diaschisis or Transtentorial Diaschisis)}

CCD was first reported by Baron et al. ${ }^{4}$ who observed a parallel reduction in blood flow and oxygen uptake in the cerebellar hemisphere contralateral to the side of supratentorial ischemic infarction. Needless to say, cerebellar hemispheres are normal on standard CT or MRI. Since its first description, CCD has been confirmed by many other groups. ${ }^{5-8}$ PET studies confirm a matching decrease in both CeBF and cerebellar oxygen consumption affecting the cerebellar hemisphere contralateral to the cerebral infarct with no change in the cerebellar oxygen extraction fraction, indicating that the reduction in CeBF is secondary to metabolic depression. ${ }^{9}$ Adding weight to these affirmations is the rapid development of CCD occurring among patients receiving intracarotid sodium amytal injections and following temporary balloon occlusion of the internal carotid artery. Prompt resolution of CCD results when either procedure is discontinued. ${ }^{10-12}$ The rapid onset and resolution of CCD proves that it is a functional transsynaptic decrease of cerebellar excitation without structural abnormality. ${ }^{13}$ Similar phenomena have been reported under other clinical circumstances, including intracranial tumors, arteriovenous malformations, hemorrhages, epilepsy, encephalitis, hydrocephalus, Moyamoya disease, Sturge-Weber syndrome, tumors, and even during Wada's test. ${ }^{6.14 .15}$ A recent study showed that it could also occur with vascular and mixed dementia. ${ }^{16}$

CCD is highest in frequency and severity in patients with infarction in the deep MCA territory but it is also observed in subjects with cortical infarcts, and the more extensive the cortical lesion, the greater the depression of metabolism in the contralateral cerebellum. ${ }^{5}$ However, others have not found any relationship to infarct size. ${ }^{7,8}$ The general lack of correlation between diaschisis, infarct volume and degree of clinical (motor and non-motor) deficit may be surprising, but the most likely explanation for this is that small deep lesions may be as effective as large cortical lesions in producing diaschisis because of their effect on tightly packed pathways. ${ }^{6-8.17-19}$

Although it may persist or even worsen, some CCD often resolves with time. The mechanisms by which cerebellar metabolism returns to normal are unknown.

CCD follows functional damage to cortical projections to the cerebellum via the cortico-ponto-cerebellar tract. ${ }^{20.21}$ Loss of excitatory influences, which originate in the contralateral cerebral cortex and relay in the pontine nuclei before reaching the cerebellar granule cells, would explain this remote transneuronal metabolic depression. ${ }^{22}$ Morphologically evident atrophy has been reported in a few cases of CCD (due to longterm diseases), a phenomenon now called crossed cerebellar atrophy. ${ }^{23}$

It is interesting to mention that glucose metabolism in the posterior fossa in cases of unilateral supratentorial tumors is diminished not only in the cerebellar cortex but also in the ipsilateral pons where synapses of the cortico-ponto-cerebellar tract are located. ${ }^{24}$ Moreover, unilateral brain stem infarction in the upper pons (but not in those with lesions below this level) was shown to cause significant contralateral cerebellar hypoactivity and confirmed that functional damage to the cortico-ponto-cerebellar pathways is the key event in the genesis of CCD. ${ }^{25-31}$

There is now evidence that in unilateral supratentorial stroke patients with crossed cerebellar hypoperfusion, the decreased metabolic rate of oxygen and reduced cerebellar blood flow were negatively correlated with the metabolic rate of oxygen in the pons, also supporting the view that interruption of the cortico-ponto-cerebellar tract is the mechanism of crossed cerebellar hypoperfusion. ${ }^{32,33}$

Usually, this cortico-cerebellar diaschisis does not lead to significant clinical features (i.e., there are no frank cerebellar signs). Rather subtle cerebellar signs, minor dyspraxia or gait ataxia may be found..$^{5,9.14}$ Lesions of predominantly excitatory cortico-pontine-cerebellar projections, most of which originate from the frontal and parietal cortices, have long been thought to be implicated in the genesis of gait apraxia. ${ }^{34}$ Cases of homolateral arm ataxia and crural paresis associated with infarction of the ACA territory (involving the upper part of the corona radiata adjacent to the lateral ventricle and in the paraventral area corresponding to the subcortical part of the ACA territory) have been reported. ${ }^{34}$ Recent studies have demonstrated that at longterm, this remote cerebellar dysfunction does not carry any additional significant clinical deficit and most likely it simply represented part of the damage induced by the stroke itself. ${ }^{18}$

\section{Cerebello-cortical Diaschisis (or Crossed Hemispheric Diaschisis or Crossed Cerebello-cortical Diaschisis)}

Cerebello-cortical diaschisis, i.e., the reverse phenomenon of $\mathrm{CCD}$, was first reported by Broich et al., ${ }^{35}$ in a 26 -year-old patient with a right cerebellar infarction following occlusion of the right vertebral artery. Botez et al. ${ }^{36,37}$ published cases of cerebellar infarctions in which SPECT neuroimaging showed crossed cerebello-cerebral hypoperfusion (at 6 months, 5 and 16 years respectively). In addition, olivopontocerebellar degeneration and 
Friedreich's ataxia patients were also found to have various degrees of decreased HMPAO uptake in both the cerebellum and cerebral hemispheres, revealing the presence of cerebellocortical diaschisis in chronic cerebellar damage in subjects with various cerebellar disorders other than infarction. ${ }^{37}$

Later, Rousseaux and Steinling ${ }^{38}$ published 11 cases of unilateral cerebellar hemorrhage manifesting significantly reduced HMPAO uptake in the contralateral hemisphere (predominantly in the frontal region and in the lenticular nucleus of the contralateral hemisphere). Crossed cerebellar-cerebral diaschisis at the subcortical level is associated with remote hypoperfusion mostly in the basal ganglia and not the thalamus, ${ }^{36-39}$ and at the cortical level, diminution of HMPAO uptake is more pronounced in the contralateral frontal lobe and less so in the parietal lobe.

It is interesting that reduced HMPAO uptake in cerebellarcortical diaschisis generally spared the thalamus and was found mainly in the basal ganglia. Some anatomical and neurochemical facts may explain this phenomenon. Snider et al. ${ }^{40}$ noted the predominance of fastigial efferents synapsing on cells in the ventral tegmental area in cats and rats whereas projections from interpositus-dentate nuclei showed a preference for the dopaminergic substantia nigra pars compacta. Projections from the substantia nigra enter the neostriatum and pass to the frontal cortex. Dopamine release in both caudate nuclei and both substantia nigrae in response to unilateral stimulation of cerebellar nuclei in the cat was demonstrated by Nieoullon et al. ${ }^{41}$

While the corticostriate projection has excitatory effects, the nigrostriatal dopaminergic projection has been generally regarded as inhibitory. ${ }^{39.42}$ On the basis of these anatomical and physiological data, the low blood flow in basal ganglia could be explained by loss of excitatory inputs from the cortex and/or perhaps from the contralateral dentate nucleus in the cerebellum (via dopaminergic projections). The reduced HMPAO uptake in basal ganglia could therefore be the result of lack of excitation due to reduced inputs from the contralateral dentate nucleus. We can conclude that cerebellar $\Rightarrow$ basal ganglia $\triangle$ cortical diaschisis follows the neurochemical-anatomical pathways.

Reversed cerebello $\Rightarrow$ basal ganglia $\Leftrightarrow$ cortical diaschisis involves most prominently the frontal and parietal areas. This is in accordance with anatomical data showing feedback connections ${ }^{43,44}$ between the prefrontal cortex and the cerebellum as well as cerebellar connections with the parietal lobe..$^{45,46}$

Neuropsychological abnormalities suggestive of contralateral fronto-parietal neuropsychological dysfunction have been demonstrated with strictly unilateral cerebellar lesions. ${ }^{47.48}$ However, after 6 months, the neuropsychological abnormalities and reaction time became normal even if the cortico-cerebellar diaschisis persisted. ${ }^{47}$ In our cases, many years after unilateral cerebellar strokes, the cerebellar $\square$ cerebral diaschisis persisted but the neuropsychological and the neurobehavioral assessments became normal. It can be concluded that in some cases, diaschisis may be present but it is not accompanied by neuropsychological or neurobehavioral deficits. In the same line of thought, experimental lesions of the dentate nucleus in monkeys also resulted in prolongation of reaction time which had a tendency to normalize within 20 days. ${ }^{49}$ Clinical data showed the absence of neuropsychological disorders in patients with chronic unilateral cerebellar CVAs.

$\mathrm{We}^{36,37,50}$ have demonstrated that bilateral chronic cerebellar lesions interfered with the following functions: (1) visuo-spatial organization for concrete tasks, a function linked to the parictocerebellar loops; (2) programming of day-to-day activity, a function linked to fronto-cerebellar loops; and (3) speed of information processing, a well-known subcortical function.

\section{Transhemispheric Diaschisis}

The term "transhemispheric diaschisis" was first introduced in 1987, but the phenomenon had already been reported in 1964 in an article by Hoedt-Rasmussen and Skinhoj ${ }^{51}$ who observed a bilateral reduction of hemispheric blood flow in patients with unilateral cerebral infarction. They measured hemispheric blood flow by means of intracarotid injection of a bolus of radioactive inert gas and an uncollimated detector placed over each hemisphere and found hemispheric blood flow to be reduced on the "healthy" side as well as in the diseased hemisphere. They did not measure cerebral metabolism, but hypothesized that unilateral infarction decreased metabolism, and consequently resulted in lower blood flow in the contralateral hemisphere due to transneuronal depression.

Meyer et al. confirmed the reduction of CBF occurring in both hemispheres following acute unilateral cerebral infarction. ${ }^{52}$ In patients with stroke, using ${ }^{18} \mathrm{FDG}-\mathrm{ECT}$, Kuhl et al. ${ }^{53}$ showed that $\mathrm{CMR}_{\mathrm{glc}}$ in the contralateral hemisphere was moderately decreased during the first week, profoundly depressed in irreversible coma, and normal after clinical recovery. Dobkin et al. ${ }^{54}$ noted a significant reduction of CBF contralateral to a strictly unilateral ischemic infarction in 19 patients by ${ }^{18} \mathrm{FCH}_{3}$ $\mathrm{CBF}$ analysis and PET.

It appears that this form of diaschisis is dependent on the integrity of the corpus callosum. In animal experiments employing chronic corpus callosum section, Kempinsky ${ }^{55}$ found that none manifested changes in the evoked response following contralateral brain injury, while in most animals with an intact corpus callosum, a marked modification of the evoked response took place (transient depression of cortical EEG and steady potentials) on the contralateral side after brain damage, including experimental MCA occlusion. Kempinsky offered a unifying concept of diaschisis by proposing that the functional activity of remote neuronal networks is facilitated by constant afferent synaptic activation from other neuronal groups, and when the facilitator group of neurones is inactivated by a focal lesion, the neurones they facilitate become depressed. ${ }^{52,55}$

Andrews ${ }^{56}$ reviewed the entire literature on the transhemispheric diaschisis phenomenon by periods based on the time from initial injury, to elucidate its time course: hyperacute phase $(<1 \mathrm{hr})$, acute phase (1-24 hrs), subacute phase ( $1-10$ days), intermediate phase (10 days- 2 months) and chronic phase ( $>2$ months). Contralateral electrical activity, such as evoked potential amplitude, is increased in the late stages after unilateral infarction, with data from the more acute periods being inconclusive. Some studies have reported a decrease in contralateral blood flow over the first week followed by a gradual return toward baseline. Most measures of contralateral metabolism show a time course similar to blood flow, i.e., a decline followed by gradual recovery.

Andrews ${ }^{56}$ brought new insights to transcallosal (or transhemispheric) diaschisis. Using a large animal model (swine) simulating operating room brain retraction ischemia, an increase in CBF and evoked potential amplitude (obtained in the acute phase in the first 5 hours following callosal section) was 
observed contralateral to retraction. Later on, however, the diaschisis resulted in contralateral depression (which gradually resolves after weeks to months in man). With retraction following callosal section, there was no increase in CBF or evoked potential amplitude contralateral to retraction. It has been concluded that during the early stages of focal, unilateral injury, diaschisis takes the form of contralateral disinhibition (as measured by $\mathrm{CBF}$ and evoked potentials). This effect is lost following callosal section. These studies confirm the abundant transcallosal connections between both hemispheres but introduce new concepts of initial "reactive contralateral disinhibition or facilitation" followed by well-known contralateral hemispheric depression.

This form of diaschisis is translated into some clinical, neurobehavioral and neuropsychological abnormalities. Motor consequences following a CVA are not only limited to the affected side. Many studies have demonstrated abnormal motor performances in the normal side when tested simultaneously with the paretic side although no abnormalities are found when the normal side is used independently. For example, a patient with right hemiparesis will, in dexterity tests using the Purdue pegboard, be able to place 24 pegs with his normal left hand and 9 with his paretic right hand in 60 seconds if he uses his hands separately. However, when asked to perform the same tasks using both hands simultaneously, he can only place 10 or 13 pegs with his normal left hand (and 8 pegs with his paretic right hand). Hence, simultaneous movements of paretic and normal hands lead to a reduction of performance in the normal hand. This incoordination problem is thought to result from an interhemispheric disequilibrium secondary to a diaschisis phenomenon which may persist.

Rizzo and Robin ${ }^{57}$ recently published data on the bilateral effects of experimental unilateral visual cortex lesions in humans (oversensitivity to signals, response time, and useful field of view under conditions of differing attention demands). An explanation for these bilateral effects in unilateral occipital lobe lesions is that damage to interhemispheric connections along their pre-splenial course could have affected the synthesis of visual information from both hemifields. ${ }^{56-58}$

\section{Cortico-thalamic Diaschisis}

Cortico-thalamic diaschisis was initially described by Kuhl et al..$^{53}$ in patients with a CVA in which the focal lesion has interrupted or sectioned thalamo-cortical projections but spared the thalamus, diminished metabolism and perfusion can be found in the thalamus. ${ }^{59.60} \mathrm{Kuhl}$ et al. ${ }^{53}$ by means of ECT with ${ }^{8} \mathrm{FDG}$ and ${ }^{13} \mathrm{NH}_{3}$ observed ipsilateral thalamic hypometabolism in 5 patients with infarcts restricted to the MCA territory, although primary thalamic blood supply originates from the posterior cerebral artery. An experimental study confirmed that local blood flow in the thalamus decreased on the ischemic side in rats with MCA occlusion. ${ }^{60.61}$ In 14 consecutive patients with unilateral cerebral infarction, hypoperfusion of the ipsilateral thalamus was as high as $85.7 \%$ as measured by ${ }^{123}$ I-IMP SPECT. ${ }^{60}$

This secondary thalamic hypometabolism and hypoperfusion probably result from a deafferentation phenomenon. ${ }^{62}$ Theories include Wallerian degeneration of thalamic neurons efferent to the region of cortical infarction or decreased excitation of thalamic neurons secondary to a loss of afferent neurons from the region of cortical infarction or a combination of these changes. ${ }^{61}$ Ipsilateral thalamic atrophy may be clinically observed after a stroke, supposedly from retrograde transsynaptic degeneration of thalamic neurons following thalamo-cortical pathway injury. ${ }^{60,63-65}$

The clinical importance of this diaschisis is controversial and not yet fully understood. In Kuhl's 5 patients with ipsilateral hypometabolism and hypoperfusion following MCA CVA, 4 were aphasic, 3 had contralateral sensory loss and none experienced the thalamic pain syndrome. ${ }^{53}$

\section{Thalamo-cortical Diaschisis}

The role of thalamo-cortical loops underlying language disorders $^{66}$ opened the way to understanding the role of subcortical structures in human cognitive behavior.

Reversed cortico-thalamic diaschisis has been described and is simply called thalamo-cortical diaschisis. In patients with subcortical stroke, PET commonly shows reduced metabolic rates of glucose or oxygen in the ipsilateral cerebral cortex. ${ }^{67.68}$ Bilateral paramedial thalamic infarcts lead to subsequent hypoperfusion and hypometabolism in prefrontal areas, unilateral posterior thalamic infarcts can result in ipsilateral parietotemporal hypometabolism, and unilateral anterior thalamic infarcts can elicit lateral frontal hypometabolism. Moreover, unilateral electrocoagulation of the VL and the Vim (stereotaxic VL-Vim thalamotomy performed for movement disorders in 11 patients reported by Baron et al.) induces widespread but preferentially ipsilateral depression of cortical energy metabolism. ${ }^{67}$

In bilateral paramedial thalamic infarcts, the diaschisis probably reflects lesion of the thalamocortical connections with a transsynaptic effect in the neocortex. Findings in patients with stereotaxically-placed unilateral thalamic lesions confirm that damage to the thalamus itself is capable of inducing cortical hypometabolism and point to a role for cortical afferents originating from the VL-Vim in the development of thalamo-cortical diaschisis. ${ }^{67-69}$ To explain the presence of contralateral presumably indirect cortical metabolic depression and unilateral thalamic damage inducing bilateral metabolic depression, the favored hypothesis implies a transcallosal spread of ipsilateral metabolic effects, ${ }^{67,70}$ but this proposed mechanism has been challenged.

Thalamo-cortical diaschisis provides the neurobiological basis for a better understanding of language disorders, memory disturbances and attention deficits observed following thalamic lesions. In patients with unilateral thalamic infarcts, significant neocortical hypometabolism is mainly found when they present neuropsychological troubles (language, memory, neglect). ${ }^{71}$ In certain patients, predominantly frontal hypometabolism coexists with frontal type disorders but also of the amnestic type. ${ }^{72.74}$ The severity of ipsilateral fronto-parieto-cortical hypometabolism found in patients with unilateral thalamic lesions following CVA or stereotaxic thalamotomy done for tremor-control in parkinsonian patients is proportional to the neuropsychological disturbances (as evaluated by both a comprehensive standard test battery and tests more sensitive to thalamic-related deficits Wechsler memory scale, the verbal scale, Raven's progressive matrices, visuospatial and visual memory tests, standard language tests, calculation tests, differential assessment of visual and verbal memory, assessment of hemineglect in right thalamic lesions, etc...). ${ }^{67}$ 
Other studies as well have found that small thalamic infarcts can result in longterm neuropsychological abnormalities. Kim et al. have reported anterograde and retrograde memory disturbances more pronounced for the verbal aspect in a patient with a previous thalamic infarct associated with hypoperfusion in the ipsilateral fronto-temporo-parietal cortex and in the contralateral cerebellum. ${ }^{75}$

Left thalamic lesions, especially of the posterior thalamus, can determine sub-cortical aphasia ${ }^{66}$ and are significantly correlated with the severity of left parieto-temporal hypometabolism. ${ }^{76}$ Moreover, abnormal verbal fluency can follow lesions of the anterior thalamus and is correlated with the severity of left latero-frontal hypometabolism. ${ }^{76-78}$ Metter et al., ${ }^{76}$ using PET technology, have shown both a direct and an indirect (through frontal hypometabolism) role of structural damage to the left thalamus and basal ganglia in impaired verbal fluency. A manic-like disorder with inappropriate laughs, jokes and comments, delirium and confabulations has also been reported with right paramedian infarction. ${ }^{72}$ The cortical metabolic changes seen after a unilateral thalamic lesion usually disappear progressively concomitantly with clinical reversal of the neuropsychological findings, suggesting an important synaptic reorganization capacity following thalamo-cortical deafferentation. Patients with bilateral paramedial thalamic infarcts present a clinical syndrome of apathy with loss of psychic autoactivation, with or without memory disturbances and compulsive presleep behavior (thalamic dementia). Current knowledge indicates that prefrontal hypometabolism is constantly accompanied by psychic akinesia due to the involvement of the fronto-striato-pallido-thalamo-cortical loops. It is noteworthy that bilateral thalamic infarcts manifestating chronic apathy with an amnestic syndrome are associated with definitive persistent cortical hypometabolism in the prefrontal regions. ${ }^{79}$

A syndrome of left multimodal motor and sensory hemineglect following right sub-cortical lesions can be found with ipsilateral cortical hypometabolism, particularly in the frontal and parietal regions. ${ }^{70,80.82}$ Fiorelli et al. ${ }^{80}$ suggested that in their study hemineglect was associated with widespread hypometabolism of the entire ipsilateral cortex in the context of moderately reduced metabolism of the ipsilateral thalamus and contralateral cerebral cortex. For Fiorelli et al., neglect resulted from functional depression of the subcortico-cortical network subserving attention that could be damaged at any one of its parts since hemineglect syndromes (including motor neglect syndromes) are known to develop following lesions affecting either the cerebral cortex (parietal or frontal areas), the thalamus, or the fiber pathways linking the two..$^{80,83-85}$

Although a study by Baron 68 indicated that cortical hypometabolism participates in the mechanism of neuropsychological impairment, the important and direct role of the subcortical structures should not be overlooked. Thus, significant correlations have been reported between intellectual performance and caudate metabolism in Huntington's disease and between some aspects of language dysfunction and caudate or thalamic hypometabolism in brain infarction.

\section{Basal Ganglia-cortical Diaschisis (or Basal Ganglia-cerebral Cortex Diaschisis)}

Orgogozo et al. ${ }^{86}$ first described remote effects of deep-seated hemispheric lesions in a series of 6 patients. Baron reviewed the literature regarding the effects of lesions of the basal ganglia on brain metabolism. ${ }^{87}$ Certain observations can be made from this excellent overview: (1) unilateral infarctions of the lenticular nucleus can result in hypometabolism of the homolateral cortex; (2) bilateral lenticular nuclei necrosis (almost restricted to the globus pallidus sparing nearby structures) may be associated with prefrontal hypometabolism in addition to severe striatal hypometabolism; (3) progressive supranuclear palsy (which constantly severely affects the globus pallidum in neuroimaging studies) can be found to have diffuse cerebral hypometabolism, predominantly in the basal ganglia, thalamus and prefrontal cortex; (4) Huntington's disease, characterized by major striatal hypometabolism, can show medial frontal hypometabolism (with, in addition, lateral prefrontal hypometabolism in depressive patients); (5) Wilson's disease, in addition to the severe hypometabolism of caudate and lenticular nuclei, presents thalamic and predominantly medial frontal cortical hypometabolism; (6) in non-demented patients with Parkinson's disease, significant hypermetabolism can be seen in some studies at the level of the lenticular nucleus (especially evident in hemi-parkinsonian patients) associated with frontal hypometabolism (although this latter observation is disputed).

In unilateral basal ganglia infarction, cortical metabolic depression could in fact, or partly, be due to thalamo-cortical diaschisis. In bilateral lesions of lenticular nuclei, prefrontal hypometabolism probably is the result of an anterograde transneuronal alteration following interruption at the pallidal level of the fronto-striato-pallido-thalamo-frontal loop. ${ }^{88}$ The severity of frontal hypometabolism seems to be greater if the site of interruption is at the thalamic level more than at the striatal or pallidal level. In progressive supranuclear palsy, the frontal hypometabolism is said to be due to a pallidal lesion leading to thalamic deafferentation. ${ }^{89}$

Neuropsychological and behavioral abnormalities have been associated with this type of diaschisis. In unilateral infarction of left lenticular nuclei, the severity of language disorders is positively correlated with the associated homolateral cortical hypometabolism: left parieto-temporal cortex hypometabolism with semantic language disorders and left frontal cortex hypometabolism with verbal fluency disorder. ${ }^{78}$ Temporo-parietal hypoactivity or left fronto-parietal hypoactivity can also be associated with more complex cognitive abnormalities..$^{90,91}$ Demeurisse et al. ${ }^{92}$ found in patients with deep-seated left hemiphere infarct lesions not involving the cortex that language disorders cannot be ascribed entirely to the presence of cortical diaschisis. However, the location and severity of the diaschisis seem to be related to the occurrence of aphasia and its severity.

In unilateral infarction of the right lenticular nucleus, the severity of hemispatial and motor neglect has been linked with ipsilateral cortical hypometabolism.

Patients with bilateral lenticular nuclei necrosis frequently present inertia, affective indifference and abnormalities of executive functions (psychic akinesia or athymhormia) with loss of psychic self-activation. It has been suggested that this clinical picture is due to prefrontal hypometabolism since other studies have linked similar neuropsychological and behavioral disorders with even mild prefrontal/frontal hypometabolism, as in schizophrenia (latero-frontal hypometabolism) ${ }^{93}$, in bilateral paramedian thalamic infarction with psychic akinesia (prefrontal hypometabolism), in post-traumatic psychic akinesia 
with loss of psychic self-activation, ${ }^{94,95}$ in amyotrophic lateral sclerosis patients with progressive dementia, ${ }^{96}$ and in progressive supranuclear palsy frontal syndrome. ${ }^{89}$ In Huntington's disease, the possibility of direct cortical lesions complicates the investigation of relationships between cortical metabolism with cognitive and affective disorders. ${ }^{97,98}$ In Wilson's disease, no study to date has looked into the cognitive or behavioral disorders associated with medial frontal hypometabolism. In some patients with Parkinson's disease, deficiencies of executive functions possibly related to cortical hypometabolism were observed. ${ }^{99-102}$

Metabolic depression of cortical and subcortical structures, as revealed by PET and SPECT (whole cerebral hypoperfusion apart from the infarcted area), secondary to cortical-subcortical disconnection in thalamic and striato-cortical pathways, may also play a role in the dementia associated with lacunar infarctions. ${ }^{103}$

Bogousslavsky recently reviewed the frontal stroke syndromes $^{79}$ and briefly discussed frontal lobe symptomatology due to stroke sparing anatomically the frontal cortex or white matter, mainly in 3 instances, i.e., lenticulo-capsular stroke, caudate stroke, and thalamic stroke, in which diaschisis may play a role. In lenticulo-capsular stroke, neurobehavioral changes are divided into 3 major clinical subsets: (1) lower capsular genu syndrome: acute-onset lethargia, abulia, often with confusion and memory loss; (2) bilateral pallidal-striate stroke: apathy, lack of motivation, severe loss of drive, affectivity and interest with the possibility of appropriate activation by external stimuli (psychic akinesia, or athymhormia); (3) multiple lacunar infarcts: impairment of frontal systems. In caudate strokes neurobehavioral abnormalities are: (1) in unilateral caudate head stroke: loss of drive and apathy coexisting with marked obsessive stereotypia and checking rituals, sometimes with affective disinhibition bursts, memory dysfunction, transcortical motor aphasia with rich semantic paraphasias and hypokinesia/motor extinction contralateral to the lesion; (2) in bilateral involvement: affective behavioral changes, vulgarity, impulsiveness, violent outbursts, hypersexuality, and sometimes minor criminal behavior contrasting with coexisting indifference, loss of independence and decreased self-care.

\section{Conclusion}

We have tried to review here all forms of diaschisis described to date with their possible relationship to neurobehavioral and neuropsychological findings. Cortico-cerebellar diaschisis may be implicated in gait apraxia but no frank cerebellar signs can be detected. Cerebello-cortical diaschisis could explain neuropsychological abnormalities suggestive of contralateral fronto-parietal dysfunction, especially in chronic cerebellar lesions. Transhemispheric diaschisis may explain motor incoordination of the normal limb when used simultaneously with the hemiparetic limb. Knowledge of the neurobehavioral consequences of cortico-thalamic diaschisis is still embryonic. Thalamo-cortical diaschisis is probably implicated in language and memory dysfunction in left lesions and hemineglect in right lesions, although the role of subcortical structures in these same functions should not be undermined. Finally, basal ganglia-cortical diaschisis has been linked to language and neglect abnormalities as well as psychic and motor akinesia.
Obviously, our knowledge of behavioral diaschisis is far from complete. Some forms of diaschisis have not been shown (yet) to yield any neurobehavioral impairment. Furthermore, neuropsychological abnormalities can normalize in spite of persistent diaschisis. Finally, the effects of diaschisis on cognitive behavior are sometimes difficult to differentiate from those that due to the anatomical-primary localization of lesions.

In the actual state-of-art of our knowledge, we can divide the different forms of diaschisis in relation to neurobehavior as follows: 1) forms of diaschisis which appear not to induce behavioral manifestations other than those elicited by the primary lesions, for example, cortico-cerebellar diaschisis and corticothalamic diaschisis; 2) forms in which diaschisis seems to be the cause of definite neurobehavioral symptoms: cerebello-basal ganglia cortical diaschisis, thalamo-cortical and transhemispheric diaschisis; 3) forms of diaschisis in which the behavioral effect of the primary lesion is difficult to disentangle from the possible remote effect: basal ganglia $\triangle$ cortical diaschisis.

\section{REFERENCES}

1. Stedman's Medical Dictionary. Baltimore: Williams and Wilkins, $24^{\text {th }}$ edition, $1984 ;$ p. 391.

2. Von Monakow C. Die Lokalisation im Grosshirn und der Abbau der Funktion durch kortikale Herde. Wiesbaden: J.F. Bergman, 1914: pp. 26-34. Translation by G. Harris, In: Pribam KH ed. Brain and Behavior. I: Mood States and Mind. Baltimore: Penguin, 1969: 27-36.

3. Chiles BW, Cooper PR. Current concepts: Acute spinal injury (review). N Engl J Med 1996; 334 (8): 514-520.

4. Baron JC, Levasseur M, Mazoyer B, et al. Crossed cerebellar diaschisis in human supratentorial brain infarction. Trans Am Neurol Assoc 1980; 105: 459-461.

5. Meneghetti G, Vorstrup S, Mickey B, et al. Crossed cerebellar diaschisis in ischemic stroke: a study of regional cerebral blood flow by ${ }^{133} \mathrm{Xe}$ inhalation and single photon emission computerized tomography. J Cereb Blood Flow Metab 1984; 4: 235-240.

6. Lenzi G, Frackowiak R, Jones T. Cerebral oxygen metabolism and blood flow in human cerebral ischemic infarction. J Cereb Blood Flow Metab 1982; 2: 321-335.

7. Martin WRW, Raichle ME. Cerebellar blood flow and metabolism in cerebral hemisphere infarction. Ann Neurol 1983; 14: 168176.

8. Kushner M, Alavi A, Reivich M, et al. Contralateral cerebellar hypometabolism following cerebral insult: a positron emission tomographic study. Ann Neurol 1984; 15: 425-434.

9. Pantano P, Baron JC, Samson Y, et al. Crossed cerebellar diaschisis. Brain 1986; 109: 677-694.

10. Kurthen M, Reichmann K, Linke DB, et al. Crossed cerebellar diaschisis in intracarotid sodium amytal procedures: a SPECT study. Acta Neurol Scand 1990; 81: 416-422.

11. Eckard DA, Purdy PD, Bonte F. Crossed cerebellar diaschisis and loss of consciousness during temporary balloon occlusion of the internal carotid artery. Am J Neuroradial 1992; 13: 55-57.

12. Brunberg JA, Frey KA, Horton JA et al. Crossed cerebellar diaschisis: occurrence and resolution demonstrated with PET during carotid temporary balloon occlusion. Am $J$ Neuroradiol 1992; 13: 58-61.

13. Dettmers C, Hartmann A, Rommel T, et al. Contralateral cerebellar diaschisis 7 hours after MCA-occlusion in primates. Neurol Res 1995; 17(2): 109-112.

14. Patronas NJ, Di Chiro G, Smith BH, et al. Depressed cerebellar glucose metabolism in supratentorial tumors. Brain Res 1984; 291: 93-101. 
15. Coubes $\mathrm{P}$, Baldy-Moulinier M, Zanca M, et al. Monitoring sodium Methohexital distribution with $99 \mathrm{mTc}$ HMPAO with single photon emission computed tomography during WADA test. Epilepsia 1995, 36(10): 1041-1049.

16. Flores LG, Futami S, Hiroaki H, et al. Crossed cerebellar diaschisis: analysis of iodine-123-IMP SPECT imaging. J Nucl Med 1995; 36: 399-402.

17. Miura H, Hirata K, Satoh Y, et al. Evolution of crossed cerebellar diaschisis in middle cerebral artery infarction. J Neuroimaging 1994; 4: $91-96$.

18. Bowler JV, Wade JPH, Jones BE, et al. Contribution of diaschisis to the clinical deficit in human cerebral infarction. Stroke 1995; 26: 1000-1006.

19. Infeld B, Davis SM, Lichtenstein M, et al. Crossed cerebellar diaschisis and brain recovery after stroke. Stroke 1995; 26: 9095.

20. Feeney DM, Baron JC. Diaschisis. Stroke 1986; 17: 817-830.

21. Meyer JS, Katsuyuki O, Muramatsu K. Diaschisis (review). Neurol Res 1994; 15: 362-366.

22. Allen GI, Tsukahara N. Cerebrocerebellar communication systems. Physiol Rev 1974; 54: 957-1006.

23. Tien RD, Ashdown BC. Crossed cerebellar diaschisis and crossed cerebellar atrophy: correlation of MR findings, clinical symptoms and supratentorial diseases in 26 pts. Am J Radiol 1992; 158: $1155-1159$.

24. Fulham MJ, Brooks RA, Hallett M, et al. Cerebellar diaschisis revisited: pontine hypometabolism and dentate sparing. Neurology 1992; 42: 2267-2273.

25. Fazekas F, Payer F, Valetitsch $\mathrm{H}$, et al. Brain stem infarction and diaschisis: a SPECT cerebral perfusion study. Stroke 1993; 24: 1162-1166.

26. Rousseaux M, Steinling M, Mazingue A, et al. Cerebral blood flow in lateral medullary infarcts. Stroke $1995 ; 26: 1404-1408$.

27. Blazquez L, Maurel G, Mensch B, et al. Diaschisis frontal ipsilateral et cerebelleux contralateral en rapport avec un infarctus pontique unilateral. Rev Neurol (Paris) 1995; 151(2): 132-135.

28. Sakai F, Aoki S, Kan S, et al. Ataxic hemiparesis with reductions of ipsilateral cerebellar blood flow. Stroke 1986; 17: 1016-1018.

29. Perani D, Di-Piero V, Lucignani G, et al. Remote effects of subcortical cerebrovascular lesions: a SPECT cerebral perfusion study. J Cereb Blood Flow Metab 1988; 8: 560-567.

30. Bowler JV, Wade JPH. Ipsilateral cerebellar diaschisis following pontine infarction. Cerebrovasc Dis 1991; 1: 58-60.

31. Perani D, Lucignani G, Pantano $P$, et al. Cerebellar diaschisis in pontine ischemia. A case report with single-photon emission computerized tomography. J Cereb Blood Flow 1987; 7(1): 127 131.

32. Yamauchi H, Fukuyama H, Kimura J. Hemodynamic and metabolic changes in crossed cerebellar hypoperfusion. Stroke 1992; 23: 855-860.

33. Attig E, Capon A, Demeurisse G, et al. Remote effect of deep-seated vascular brain lesions on cerebral blood flow. Stroke 1990; 21: 1555-1561.

34. Meyer JS, Barron DW. Apraxia of gait: a clinicophysiological study. Brain 1960; 83: 261-284.

35. Broich K, Hartmann A, Biersack HJ, et al. Crossed cerebello-cerebral diaschisis in a patient with cerebellar infarction. Neurosci Lett 1987; 83: 7-12.

36. Botez MI, Leveille J, Botez Th. Role of the cerebellum in cognitive thought: SPECT and neuropsychological findings. Proceedings of the $13^{\text {th }}$ Brain Impairment Conference. The University of Sydney, Australia, 2-4 Sept. 1988.

37. Botez MI, Leveille J, Lambert R, et al. Single photon emission computed tomography (SPECT) in cerebellar disease: cerebellocerebral diaschisis. Eur Neurol 1991; 31: 405-412.

38. Rousseaux M, Steinling M. Crossed hemispheric diaschisis in unilateral cerebellar lesions. Stroke 1992; 23: 511-514.

39. Sommezoglu K, Sperling B, Henricksen T, et al. Reduced contralateral hemispheric flow measured by SPECT in cerebellar lesions: crossed cerebral diaschisis. Acta Neurol Scand 1993; 87: 275-280.

40. Snider RA, Maiti A, Snider SR. Cerebellar pathways to ventral midbrain and nigra. Exp Neurol 1976; 53: 714-728.
41. Nieoullon A, Cheramy A, Glowinski J. Release of dopamine in both caudate nuclei and both substantiae nigrae in response to unilateral stimulation of cerebellar nuclei in the cat. Brain Res 1978; 148: 143-152.

42. Côté L, Crutcher MD. The basal ganglia. In: Kandel ER, ed. Principles of Neural Science. New York: Elsevier, 1985: 523534.

43. Schmahmann JD, Pandya DN. Topographic organization of the motor cortex projections to pons in the rhesus monkey. Soc Neurosci Abstr 1995; 21: 659.

44. Middleton FA, Strick PL. Anatomical evidence for cerebellar and basal ganglia involvement in higher cognitive function. Science 1994; 266: 458-461.

45. Sasaki K, Oka H, Matsuda Y, et al. Electrophysiological studies of the projections from the parietal association area to the cerebellar cortex. Exp Brain Res 1975; 23: 91-102.

46. Glickstein M, May JG, Mercier BE. Corticopontine projections in the macaque: the distribution of labelled cortical cells after large injections of horseradish peroxidase in the pontine nuclei. $J$ Comp Neurol 1985; 235: 343-359.

47. Botez-Marquard T, Léveillé J, Botez MI. Neuropsychological functioning in unilateral cerebellar damage. Can J Neurol Sci 1994; 21: 353-357.

48. Silveiri MC, Leggio MG, Molinari M. The cerebellum contributes to linguistic production: a case of agrammatic speech following a right cerebellar lesion. Neurology 1994; 44: 2047-2050.

49. Amrani K, Pellerin JP, Lamarre Y. The effect of bilateral lesions of the cerebellar nuclei on motor performance in monkey. Soc Neurosci Abstr 1988; 13: 1239.

50. Botez MI. The cerebellum. In: Ramachandran S, ed. Encyclopedia of Human Behavior, San Diego: Academic Press, 1994; 1: 549 560.

51. Hoedt-Rasmussen K, Skinhoj E. Transneural depression of the cerebral hemispheric metabolism in man. Acta Neurol Scand 1964; 40: 41-46.

52. Meyer JS, Shinohara Y, Kanda T, et al. Diaschisis resulting from acute unilateral cerebral infarction: quantitative evidence for man. Arch Neurol 1970; 23: 241-247.

53. Kuhl DE, Phelps ME, Kowell AP, et al. Effects of stroke on local cerebral metabolism and perfusion; mapping by emission computed tomography of ${ }^{18} \mathrm{FDG}$ and ${ }^{3} \mathrm{NH}_{3}$. Ann Neurol 1980; 8: 47-60.

54. Dobkin JA, Levine RL, Lagreze HL, et al. Evidence for transhemispheric diaschisis in unilateral stroke. Arch Neurol 1989; 46: 1333-1336.

55. Kempinsky WH. Experimental study of distant effects of acute focal brain injury: study of diaschisis. Arch Neurol Psychiatry 1958; 79: 376-389.

56. Andrews RJ: Transhemispheric diaschisis. A review and comment. Stroke 1991; 22: 943-949.

57. Rizzo M, Robin DA. Bilateral effects of unilateral visual cortex lesions in human. Brain 1996; 119: 951-953.

58. Buchkremer-Ratzmann I, Matthias A, Hagemann G, et al. Electrophysiological transcortical diaschisis after cortical photothrombosis in rat brain. Stroke 1996; 27: $1105-1111$.

59. Wise RJS, Bernardi S, Frackowiak RSJ, et al. Serial observations on the pathophysiology of acute stroke: the transition from ischemia to infarction as reflected in regional oxygen extraction. Brain 1983; 106: 197-222.

60. Sakashita Y, Hiroshi M, Kiyoshi K, et al. Hypoperfusion and vasoreactivity in the thalamus and cerebellum after stroke. Stroke 1993; 24: 84-87.

61. Tamura A, Graham DI, McCulloch J, et al. Focal cerebral ischaemia in the rat: 2 . Regional cerebral blood flow determined by $\left[{ }^{14} \mathrm{C}\right]$ iodoantipyrine autoradiography following middle cerebral artery occlusion. J Cereb Blood Flow Metab 1981; 1: 61-69.

62. Heiss WD, et al. Decreased glucose metabolism in functionally inactivated brain regions in ischemic stroke and its alteration by activating drugs. In: Meyer JS, Lechner $\mathrm{H}$, Reivich $\mathrm{M}$, Oh EO, eds. Cerebral Vascular Diseases 4. Amsterdam: Excepta Medica, $1984 ; 162-167$.

63. Tamura A, Tahira Y, Nagashima $\mathrm{H}$, et al. Thalamic atrophy following cerebral infarction in the territory of the middle cerebral artery. Stroke 1991; 22: 615-618. 
64. Fujie W, Kirino T, Tomukai N, et al. Progressive shrinkage of the thalamus following middle cerebral artery occlusion in rats. Stroke 1990; 21: 1485-1488.

65. Kataoka K, Hayakawa T, Yamada K, et al. Neuronal network disturbance after focal ischemia in rats. Stroke 1989; 20: 12261235.

66. Botez MI, Barbeau A. Role of subcortical structures, and particularly of the thalamus in the mechanisms of speech and language. Int J Neurol 1971; 8: 300-320.

67. Baron JC, Levasseur M, Mazoyer B, et al. Thalamocortical diaschisis: positron emission tomography in humans. J Neurol Neurosurg Psychiatry 1992; 55: 935-942.

68. Baron JC. Depression of energy metabolism in distant brain structures: studies with positron emission tomography in stroke patients. Semin Neurol 1989; 4: 281-285.

69. Pappata S, Mazoyer B, Tran Dinh S, et al. Effects of capsular or thalamic stroke on metabolism in the cortex and cerebellum: a positron tomography study. Stroke 1990; 21: 519-524.

70. Baron JC, D'Antona R, Pantano P, et al. Effects of thalamic stroke on energy metabolism of the cerebral cortex. A positron tomography study in man. Brain 1986; 106: 1243-1259.

71. Baron JC, D'Antona R, Serdaru M, et al. Hypometabolisme cortical apres lesion thalamique chez l'homme: études par la tomographie à positon. Rev Neurol 1986; 142: 456-474.

72. Bogousslavsky J, Ferrassini M, Regli F, et al. Manic delirium and frontal-like syndrome with paramedian infarction of the right thalamus. J Neurol Neurosurg Psychiatry 1988; 51: 116-119.

73. Rousseaux M, Kassiotis P, Signoret JL, et al. Syndrome amnesique par infarctus restreint du thalamus anterieur droit. Rev Neurol 1991: 147: 809-818.

74. Sandson TA, Daffner KR, Carvalho PA, et al. Frontal lobe dysfunction following infarction of the left-sided medial thalamus. Arch Neurol 1991; 48: 1300-1303.

75. Kim JS, Lee JH, Suh DC, Lee MC. Spectrum of lateral medullary syndrome: correlation between clinical findings and magnetic resonance imaging in 33 subjects. Stroke 1994; 25: 1405-1410.

76. Metter EJ, Riege WH, Hanson WR, et al. Correlation of glucose metabolism and structural damage to language function in aphasia. Brain Lang 1984; 21: 187-207.

77. Metter EJ, Wasterlain CG, Kuhl DE, et al. ${ }^{18}$ FDG PET in a study of aphasia. Ann Neurol 1981; 10: 173-183.

78. Karbe H, Szelies B, Herholz K, et al. Impairment of language is related to left parieto-temporal glucose metabolism in aphasic stroke patients. J Neurol 1990; 237: 19-23.

79. Bogousslavsky J. Frontal stroke syndromes (review). Eur Neurol 1994; 34: 306-315.

80. Fiorelli M, Blin J, Bakchine S, et al. PET studies of cortical diaschisis in patients with motor hemi-neglect. J Neurol Sci 1991; 104: 135-142.

81. Perani D, Vallar S, Messa C, Fazio F. Aphasia and neglect after subcortical stroke. A clinical cerebral perfusion correlation study. Brain 1987; 110: 1211-1229.

82. Bogousslavsky J, Miklossy J, Regli F, et al. Subcortical neglect: neuropsychological, SPECT, and neuropathological correlations with anterior choroidal artery infarction. Ann Neurol 1988; 23: 448-452.

83. Metter EJ, Riege WH, Hanson WR, et al. Subcortical structures in aphasia. Arch Neurol 1988; 45: 1229-1234.

84. Mesulam MM. A cortical network for directed attention and unilateral neglect. Ann Neurol 1981; 10: 309-325.
85. Heilman K M, Watson RT. Mechanisms underlying the unilateral neglect syndrome. In: Weinstein EA and Friedland RP, eds. Advances in Neurology, Vol. 18, New York: Raven Press, 1986.

86. Orgogozo JM, Larsen B, Skyhoj T, et al. Evidence of cortical disconnection in deep hemispheric strokes as revealed by rCBF. Acta Neurol Scand 1979; 60 (Suppl 72); 258-259.

87. Baron JC. Consequences des lesions des noyaux gris centraux sur l'activite metabolique cerebrale: implications cliniques. Rev Neurol (Paris) 1994; 150(8-9): 599-604.

88. Laplane D, Levasseur M, Pillon B, et al. Obsessive-compulsive and other behavioral changes with bilateral basal ganglia lesions. A neuropsychological, magnetic resonance imaging and positron tomography study. Brain 1989; 112: 699-725.

89. Blin J, Baron JC, Dubois B, et al. PET study in progressive supranuclear palsy: brain hypometabolic pattern and clinicometabolic correlations. Arch Neurol 1990; 47: 747-752.

90. Pasquier F, Leys D, Steinling M, et al. Agnosie auditive unilaterale droite consecutive a une hemorrhagie lenticulaire gauche. Rev Neurol (Paris) 1991; 147: 129-137.

91. Viader F, Lechevalier B, Eustache F, et al. Un cas d'aphasie avec troubles du discours par infarctus des noyaux caude et lenticulaire gauches. Rev Neurol (Paris) 1987; 143: 814-822.

92. Demeurisse G, Capon A, Verhas V, Attig E. Pathogenesis of aphasia in deep-seated lesions: likely role of cortical diaschisis. Eur Neurol 1990; 30: 67-74.

93. Joyce EM. The relevance to psychiatry of recent advances in functional imaging. J Neurol Neurosurg Psychiatry 1992; 55: 427430.

94. Bogousslavsky J, Regli F, Delaloye B, et al. Loss of psychic selfactivation with bithalamic infarction: neurobehavioural, CT, MRI and SPECT correlates. Acta Neurol Scand 1991; 83: 309316.

95. Laplane D, Dubois B, Pillon B, Baulac M. Perte d'autoactivation psychique et activite mentale stereotypee par lesion frontale. Rev Neurol (Paris) 1988; 144: 564-570.

96. Makoto T, Kondo S, Hirai S, et al. Cerebral blood flow and oxygen metabolism in progressive dementia associated with amyotrophic lateral sclerosis. J Neurol Sci 1993; 120: 22-28.

97. Martin WRW, Clark C, Ammann W, et al. Cortical glucose metabolism in Hungtington's disease. Neurology 1992; 42: 223229.

98. Berent S, Giordani B, Lehtinen S, et al. Positron emission tomographic scan investigations of Huntington's disease: cerebral metabolic correlates of cognitive function. Ann Neurol 1988; 23: $541-546$.

99. Martin WRW, Beckman JH, Calne DB, et al. Cerebral glucose metabolism in Parkinson's disease. Can J Neurol Sci 1984; 11: 169-173.

100. Baron JC. Lesions des noyaux gris centraux: consequences sur l'activite metabolique cerebrale. Rev Neurol 1994; 150: 599604.

101. Mohr E, Mann UM, Miletich RS, et al. Neuropsychological and glucose metabolic profiles in asymmetric Parkinson's disease. Can J Neurol Sci 1992; 19: 163-169.

102. Marie RM, Rioux P, Travere JM, et al. Working memory and cerebral glucose metabolism $\left(\mathrm{CMR}_{\mathrm{glc}}\right)$ in Parkinson's disease using PET. Neurology 1993; 43: 549.

103. Loeb C. Dementia due to lacunar infarctions: a misnomer or a clinical entity. Eur Neurol 1995; 35: 187-192. 